\title{
Photoactivity, reversibility, and stability of a merocyanine-type photoacid in polymer films
}

\author{
Nawodi Abeyrathna and Yi Liao* \\ Department of Chemistry, Florida Institute of Technology, Melbourne, Florida 32901, United States
}

\begin{abstract}
Over the past years, merocyanine type photoacids have been used for the development of various visible-light responsive polymers and polymer sensors. However, the physicochemical behavior of this type of photoacid in polymers has not been studied previously. In this work, the photoactivity, reversibility, and stability of a merocyanine-type photoacid in polymer films are investigated. Polymer films doped with the photoacid were found to be stable under ambient conditions over half a year. Kinetics of the reaction is complicated for both the forward and backward process due to slow proton diffusion in the polymer. Improving proton transfer by addition of ammonium trifluoromethanesulfonate enhances the reverse reaction rate. The photoacid-doped polymer showed good photostability upon a test of 100 cycles of irradiation and recovery in the dark.
\end{abstract}




\section{Introduction}

Recently discovered merocyanine-type photoacid can reversibly change large magnitude of proton concentration using visible light [1]. The applications of this type of photoacid in controlling proton transfer in chemical, material and biological processes has been reported over the past few years [2-8]. This type of photoacid has a protonated merocyanine (MEH) structure. (Fig. 1) It is related to the photochromic spiropyran (SP), which have been extensively studied [9]. A spiropyran undergoes a ringopening reaction under UV light and transforms to a phenolate merocyanine (ME). A merocyanine-type photoacid undergoes a cyclization reaction under visible light to form, formally, a protonated spiropyran (SPH), which is acidic and releases a proton. The acidic state is metastable and thermally relaxes back to MEH in the dark.

Merocyanine type photoacid is a useful tool for developing photoresponsive polymers and several applications in this field have been demonstrated. For example, Chumbimuni-Torres and coworkers utilized some merocyanine-type photoacids to develop ion-sensing polymer membranes [5]. Maity et al. showed that the rate of formation and mechanical properties of a hydrogel can be controlled by photoinduced protonation using this type of photoacid [3]. Our group showed that proton concentration generated by a merocyanine-type photoacid can change the volume of polyacrylamide hydrogel [1], the conductivity of polyaniline [10] and color of polymer films doped with $\mathrm{pH}$ indicators [7]. Although properties of merocyanine-type photoacids in solutions have been reported previously [11], their physicochemical properties in solid polymer materials have not been studied in detail. Understanding their behaviors in solid polymer materials is critical for developing photoresponsive materials based on these photoacids. Herein, we report our study on the photoactivity, reversibility, and stability of merocyanine-type photoacids in polymer films. 


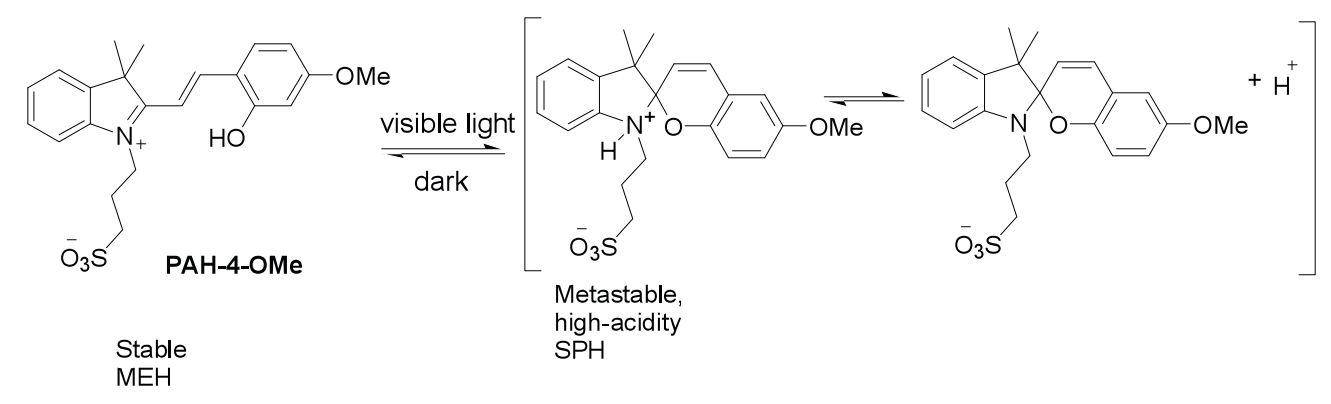

Fig. 1. Reversible photoreaction of a merocyanine-type photoacid.

\section{Results and discussion}

In this work, polymer films doped with a photoacid (PAH-4-OMe in Fig. 1) are studied. PAH-4-OMe has been previously reported for the development of an odorant-releasing material [6]. It is chosen for this study due to its relatively fast reverse reaction comparing to the one without an OMe group. The reverse reaction of merocyanine-type photoacids in polymers is normally quite slow. Using PAH-4OMe allows us to study its reverse reaction as well as photostability as described later. Thin films of the PAH-4-OMe was prepared by spin casting solutions of the photoacid and poly(hydroxyethyl methacrylate) (PHEM) on glass substrates. Poly(hydroxyethyl methacrylate) (PHEM) is a methacrylate polymer containing hydroxyl groups on flexible side chains, which mimic the environment of an alcohol and may assist proton transfer in the polymer. The weight ratio of the photoacid to PHEM was $2.5 \%$. Methanol solutions of the mixture ( $5 \mathrm{wt} \%$ in methanol) were casted on glass slides first at $500 \mathrm{rpm}$ for $30 \mathrm{~s}$ then $1000 \mathrm{rpm}$ for $1 \mathrm{~m}$. The thin films samples were baked on a hot plate for $5 \mathrm{mins}$ at $70{ }^{\circ} \mathrm{C}$, then kept under vacuum at room temperature for 3 days. The resultant orange films were transparent and homogenous. They were very stable under ambient conditions and showed essentially the same photochemical properties after half a year. 
The photoreaction of PAH-4-OMe in PHEM was monitored by UV-Vis spectroscopy during irradiation. The polymer film on a glass substrate was kept in a tilted position, and the irradiation was perpendicular to the detecting beam (Fig. 2). The light source was a light emitting diode (LED) array with a wavelength at $470 \mathrm{~nm}$. The light intensity on the film was measured by a quantum meter and the photon

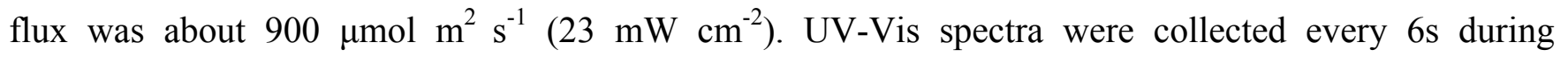
irradiation (Fig. 2).
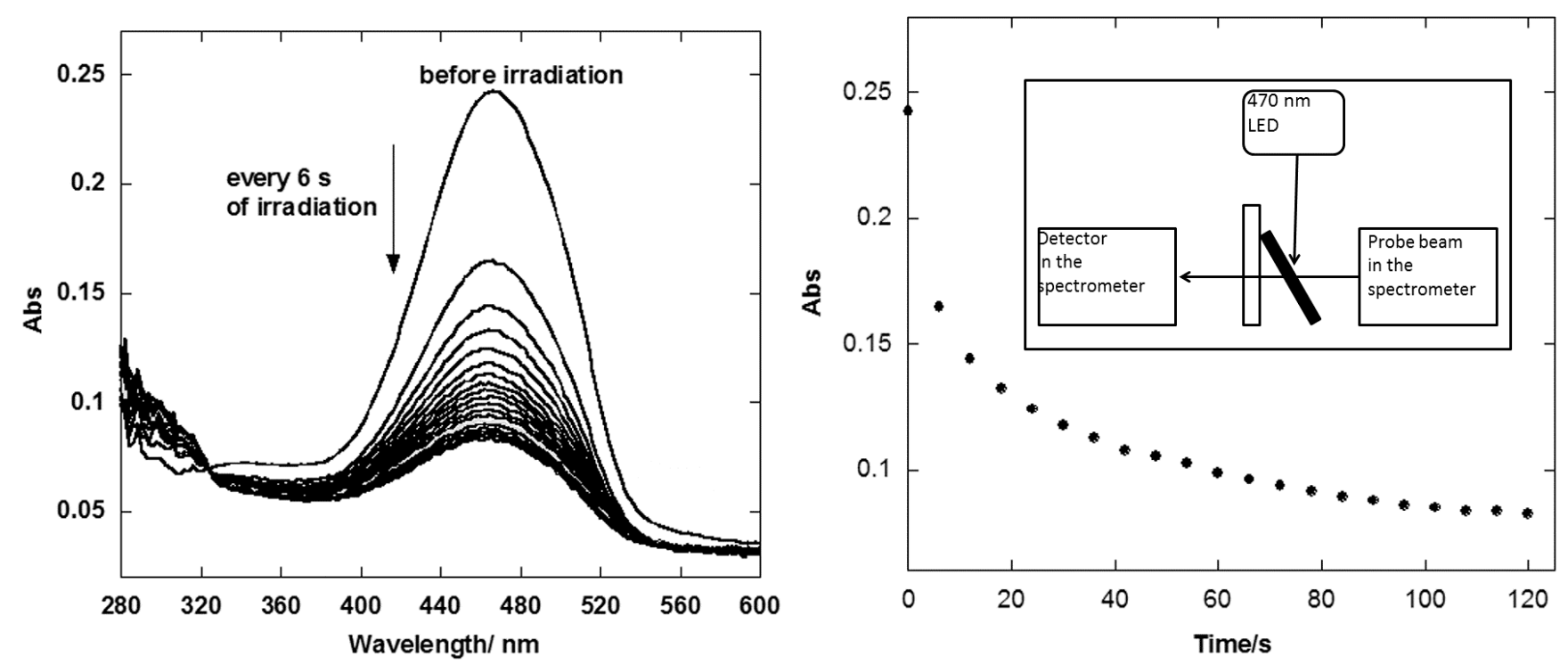

Fig. 2. UV-Vis spectra of photoacid 2-polymer film upon irradiation with $470 \mathrm{~nm}$ LED light source collected every $6 \mathrm{~s}$ (left) and change of absorbance of MEH form upon irradiation.

The MEH form of PAH-4-OMe has an absorption maximum $\left(\lambda_{\max }\right)$ at $466 \mathrm{~nm}$ in the polymer film. As shown in Fig. 2, upon irradiation, absorbance at $466 \mathrm{~nm}$ rapidly decreased in the first 6 second. Then the reduction of the absorbance quickly slowed down. After about 90 second, only marginal but still differentiable reduction was observed every 6 second. After 2 mins, the absorbance was reduced to about $66 \%$ of the original level. A clear isosbestic point at $320 \mathrm{~nm}$ was observed. Although the 
photoreaction is formally a unimolecular reaction, the data obtained here cannot be fit to first order kinetic equations. The rate of the reverse reaction must be comparable to that of the photoreaction. The process is further complicated by proton diffusion from the photoacid to the polymer. Proton diffuses much slower in a polymer than in a protic solution. Upon irradiation, protons are released from the photoacid (SP form), which cannot diffuse quickly to the whole polymer. Therefore, a local concentration of proton was built up around SP. Previous work showed that the rate-limiting step of the reverse reaction is the recombination of SP and $\mathrm{H}^{+}$, and the reaction follows a $2^{\text {nd }}$ order kinetics [11]. In polymer, the concentration of the photoacid is high. In fact, $2.5 \mathrm{wt} \%$ is equal to approximately $60 \mathrm{mM}$ comparing to $\sim 0.5 \mathrm{mM}$ commonly used for solution study. A high concentration of the photoacid resulted in a high concentration of $\mathrm{SP}$ and $\mathrm{H}+$ at the late stage of the photoreaction, which lead to a fast reverse reaction and slow overall reaction. In addition, the local concentration due to slow diffusion in polymer could be higher or lower than the average value, resulting in complicated kinetics.

The reverse reaction was studied by monitoring the recovery of the absorbance at $466 \mathrm{~nm}$ after irradiation. A photoacid-doped polymer film was irradiated with the $470 \mathrm{~nm}$ LED light source for 8 mins. Then UV spectra were taken while the sample was kept in the dark. In the first 20 mins, the sample was scanned every min. (Fig. 3) Since the reverse reaction became very slow after 20 mins, the time interval was increased to 10 mins and after 10 scans, it was further increased to one hour for the next 5 scans (Fig. 3). A rapid increase of the absorbance was observed within first 20 mins and the rate slowed down with time. Unlike the reverse reaction in solutions [11], the data cannot be fitted into either $1^{\text {st }}$ order or $2^{\text {nd }}$ order rate equation. We also tried to fit only the data in the first $20 \min$ to $1^{\text {st }}$ and $2^{\text {nd }}$ order rate equation and did not succeed. Fig. 3 shows that, $59 \%$ of the MEH form recovered after 20 min, and it took $180 \mathrm{~min}$ for $90 \%$ recovery. UV irradiation (254 $\mathrm{nm}$ and $300 \mathrm{~nm}$ ) was used to increase 
the reverse reaction rate. However, the UV irradiated sample did not show a faster reverse process than that of a non-irradiated sample.
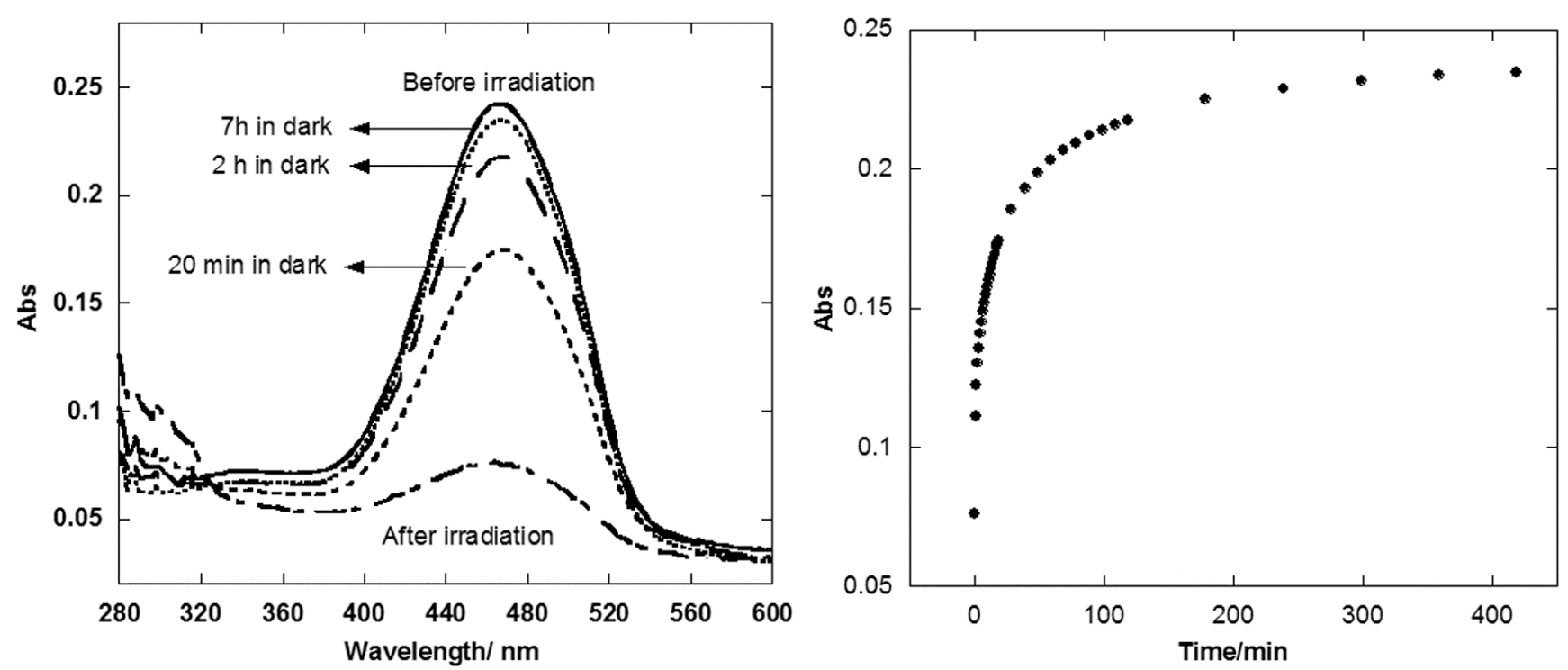

Fig. 3. UV-Vis spectra of PAH-4-OMe in PEHM film in the dark after irradiation (left) and change of absorbance at $466 \mathrm{~nm}$ form after irradiation.

The reversibility of the photoacid in polymer film was tested by repeated exposure to $470 \mathrm{~nm}$ irradiation. The sample was irradiated for $5 \mathrm{mins}$ and then kept in the dark for 10 min for each cycle. The change of MEH was monitored by UV-Vis absorbance at $466 \mathrm{~nm}$. (Fig. 4) After the first irradiation, $76 \%$ of MEH reacted. Keeping the sample in the dark for 10 min resulted in $42 \%$ of the photoacid at the $\mathrm{MEH}$ form. In the following cycles, both the percentage of $\mathrm{MEH}$ after each irradiation and that after recovery in the dark slightly decreased with the number of cycles. After about 5 cycles, the percentage change for both irradiating and recovery processes stabilized to $\sim 32 \%$. After the $10^{\text {th }}$ irradiation, the sample was kept in the dark for $24 \mathrm{~h}, 95 \%$ of $\mathrm{MEH}$ recovered. These results show that the photoacid has good reversibility and stability in the polymer film. 

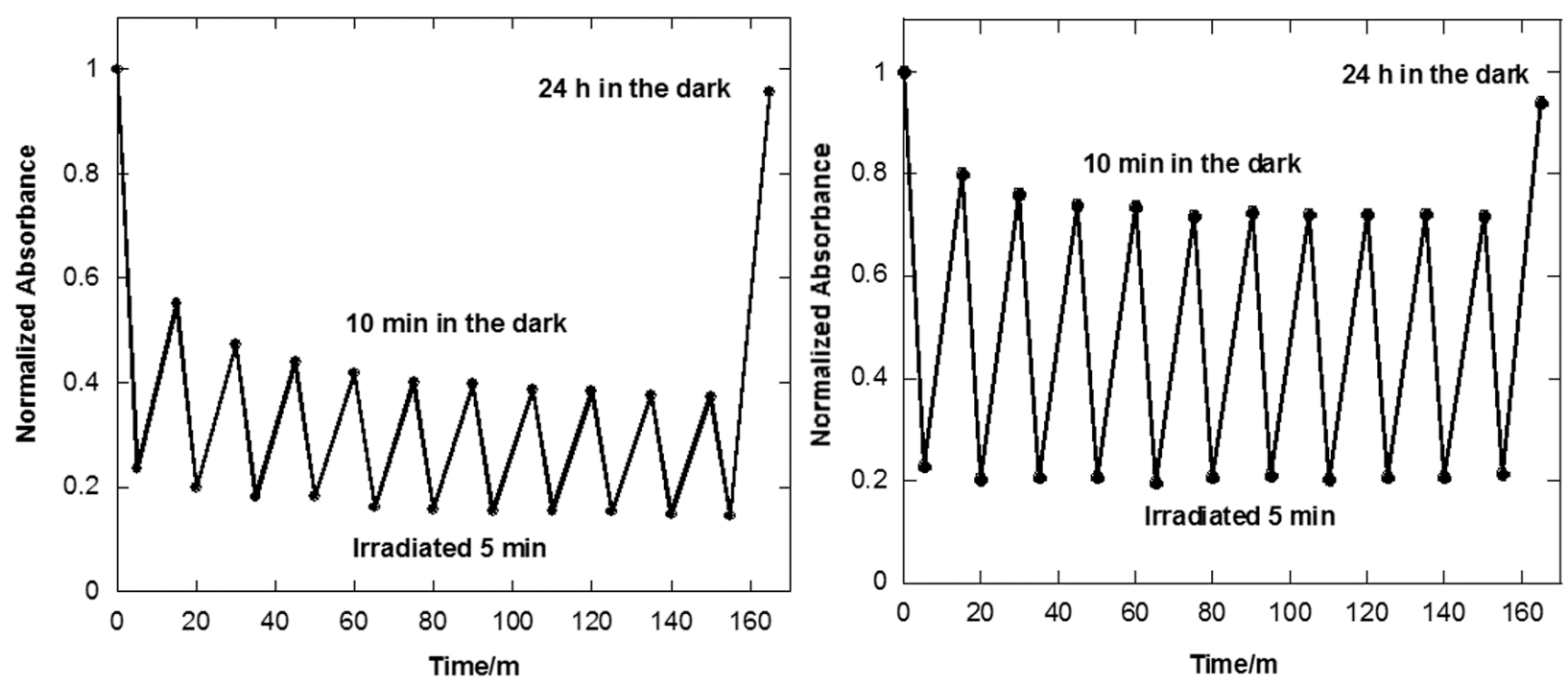

Fig. 4. Normalized absorbance of PAH-4-OMe in PHEM film (left), and PHEM film with 5\% of ATFMS (right) at $466 \mathrm{~nm}$ under repeated irradiating and recovery in the dark (details in the text).

For many applications related to photoswitching, a fast reverse process is required. As described above, keeping the sample in the dark for 10 min only resulted in $42 \%$ of recovery. Slow proton diffusion in the polymer film is one of the major reasons for the slow reverse reactions. The reverse reaction needs proton to reform MEH. Although the initial rate for the reverse reaction could be fast due to high local proton concentration, the overall rate is slow because the protons that have diffused in the polymer cannot move back fast. In order to prove this hypothesis and enhance the reaction rate, ammonium trifluoromethanesulfonate (ATFMS) was added. The ammonium cation of ATFMS can act as a proton donor for hydrogen bonds [12], and the triflate anion can accept protons. In fact, ATFMS has been used as cathode additive in fuel cell systems to improve the proton conductance [13]. Also other triflate salts have been used to increase proton and charge transfer in polyaniline [14]. Therefore $5 \mathrm{wt} \%$ of ATFMS was added to the polymer film. The sample was subjected to 10 cycles of 5 min irradiation and $10 \mathrm{~min}$ in dark. (Fig. 4) About $74 \%$ of the absorbance for MEH form was recovered after the $1^{\text {st }}$ cycle 
comparing to $42 \%$ for the sample without ATFMS. The change of the absorbance in the following cycles was about the same. After 10 cycles, the sample was kept in the dark for $24 \mathrm{~h}$, about $94 \%$ of MEH was recovered.

Photostability of the photoacid in the polymer film with $5 \mathrm{wt} \%$ of ATFMS was evaluated by subjecting a sample to 100 cycles of 3-min irradiation and 5-min in the dark per cycle (Fig. 5). As the previous tests, the experiment was performed under ambient conditions without any special caution. After the film was tested for 50 cycles it was kept in the dark overnight ( $16 \mathrm{~h})$, at which point $92 \%$ of MEH was recovered. Then the next 50 cycles were performed. Similar behaviors as the previous tests were observed. After 100 cycles, the sample was kept in the dark for $16 \mathrm{~h}$, and $90 \%$ of MEH recovered, which indicates good photostability of the photoacid.

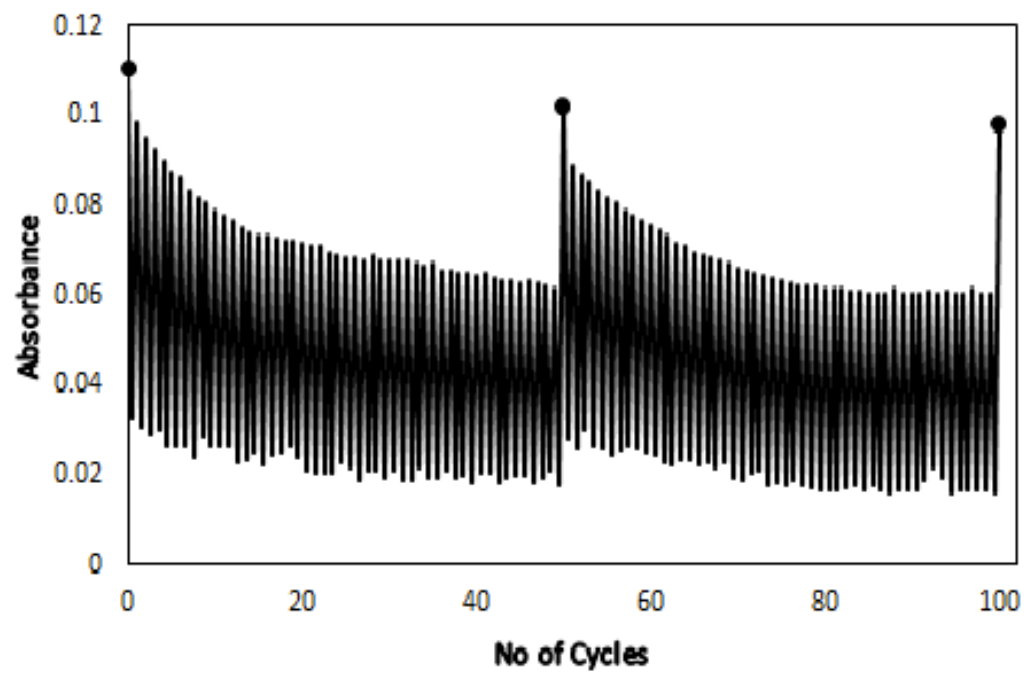

Fig. 5. Absorbance of PAH-4-OMe in PHEM film with 5\% of ATFMS subjecting a sample to 100 cycles of 3-min irradiation and 5-min in dark per cycle. 


\section{Conclusion}

Photoreactions and stability of a merocyanine-type photoacid in poly(hydroxyethyl methacrylate) films have been investigated. The polymer films doped with the photoacid were found to be stable under ambient conditions after half a year. Kinetics is complicated for both the forward and backward process due to slow proton diffusion in the polymer. Improving proton transfer by addition of ATFMS enhances the reversibility. The photoacid-doped polymer showed good photostability during a test of 100 cycles of irradiation and recovery in the dark.

\section{Acknowledgement}

Support from National Science Foundation is gratefully acknowledged.

\section{References}

[1] Z. Shi, P. Peng, D. Strohecker, Y. Liao, J. Am. Chem. Soc. 133 (2011) 14699-14703; N. Abeyrathna, Y. Liao. J. Am. Chem. Soc. 137 (2015) 11282.

[2] C. Maity, W. E Hendriksen, J. H. Van Esch, R. Eelkema, Angew. Chem. Int. Ed. 54 (2015) 998 1001.

[3] L. A. Tatum, J. T. Foy, I. Aprahamian, J. Am. Chem. Soc. 136 (2014) 17438-17441.

[4] H. Bao, F. Li, L. Lei, B. Yang, Z. Li, RSC Adv. 4 (2014) 27277-27280.

[5] V. K Johns, P. K. Patel, S. Hassett, P. Calvo-Marzal, Y. Qin, K. Y. Chumbimuni-Torres, Anal. Chem. 86 (2014) 6184-6187; P. K. Patel, K. Y Chumbimuni-Torres, Analyst. 141 (2016) 85-89. 
[6] Z. Wang, V. K. Johns, Y. Liao, Chem. Eur. J. 20 (2014) 14637-14640.

[7] H. Chen, Y. Liao, J. Photochem. Photobiol. A: Chem. 300 (2015) 22-26.

[8] Y. Luo, C. Wang, P. Peng, M. Hossain, T. Jiang, W. Fu, Y. Liao, M. Su, J. Mater. Chem. B. 1 (2013) $997-1001$.

[9] R. Richert, Macromolecules 21 (1998) 923-929; J. Hobley, U. Pfeifer-Fukumura, M. Bletz, T. Asahi, H. Masuhara, and H. Fukumura, J. Phys. Chem. A 106 (2002) 2265-2270; J. T. C. Wojtyk, A. Wasey, N.-N. Xiao, P. M. Kazmaier, S. Hoz, C. Yu, R. P. Lemieux, E. Buncel, J. Phy. Chem. 111 (2007) 25112516; F. M. Raymo, R. J. Alvarado, S. Giordani, M. A. Cejas, J. Am. Chem. Soc. 125 (2003) 23612364.

[10] Z. Shi, P. Peng, V. K. Johns, Y. Liao, Polym. Prepr. 53 (2012) 125-126.

[11] V. K. Johns, Z. Wang, X. Li, Y. Liao, J. Phys. Chem. A. 117 (2013) 13101-13104.

[12] I. Ahmed, M. Tong, J. W. Jun, C. Zhong, S. H. Jhung, J. Phys. Chem. C. 120 (2016) 407-415.

[13] S.-G.Hong, K. Kwon, M.-J. Lee, D. Y. Yoo, Electrochem. Commun. 11 (2009) 1124-1126.

[14] B. J. Polk, K. P.-Kamloth, M. Josowicz, J. Janata, J. Phys. Chem. B, 106 (2002) 11457-11462. 